\title{
BMJ Open Socioeconomic inequality in oral anticoagulation therapy initiation in patients with atrial fibrillation with high risk of stroke: a register-based observational study
}

\author{
Elin Danielsen Lunde (D) , , ${ }^{1,2}$ Albert Marni Joensen, ${ }^{1}$ Kirsten Fonager, ${ }^{3,4}$ \\ Søren Lundbye-Christensen, ${ }^{2,5}$ Søren Paaske Johnsen, ${ }^{6}$ Mogens Lytken Larsen, ${ }^{3}$ \\ Gregory YH Lip, ${ }^{7,8}$ Sam Riahi ${ }^{1,2,3}$
}

To cite: Lunde ED, Joensen AM, Fonager $\mathrm{K}$, et al. Socioeconomic inequality in oral anticoagulation therapy initiation in patients with atrial fibrillation with high risk of stroke: a register-based observational study. BMJ Open 2021;11:e048839. doi:10.1136/ bmjopen-2021-048839

- Prepublication history and additional supplemental material for this paper are available online. To view these files, please visit the journal online (http://dx.doi.org/10.1136/ bmjopen-2021-048839).

Received 11 January 2021 Accepted 06 May 2021

Check for updates

(C) Author(s) (or their employer(s)) 2021. Re-use permitted under CC BY-NC. No commercial re-use. See rights and permissions. Published by BMJ.

For numbered affiliations see end of article.

Correspondence to Dr Elin Danielsen Lunde; e.lunde@rn.dk

\section{ABSTRACT}

Objective The study aimed to examine the association between socioeconomic factors (SEFs) and oral anticoagulation $(\mathrm{OAC})$ therapy and whether it was influenced by changing guidelines. We hypothesised that inequities in initiation of $\mathrm{OAC}$ reduced over time as more detailed and explicit clinical guidelines were issued.

Design Register-based observational study.

Settings All Danish patients with an incident hospital diagnosis of atrial fibrillation (AF), aged $\geq 30$ years old and with high risk of stroke from 1 May 1999 to 2 October 2015 were included. Absolute risk differences (RD) (95\% Cl) were used to measure the association.

Participants 154448 patients (mean age 78.2 years, men 47.3\%).

Exposure Education, family income and cohabiting status were the SEFs used as exposure.

Outcome A prescription of OAC within -30 to +90 days of baseline (incident AF).

Results During 2002-2007, the crude RD of initiation of $\mathrm{OAC}$ for men with high education was $14.9 \%$ (12.8 to 16.9$)$. Inequality reduced when new guidelines were published, and in 2013-2016 the crude RD was 5.6\% (3.5 to 7.7). After adjusting for age, the RD substantially reduced. The same pattern was seen for cohabiting status, while inequality was smaller and more constant for income.

Conclusion Patients with low income, low education and living alone were associated with lower chance of being initiated with OAC. For education and cohabiting status, the crude difference reduced around 2011, when more detailed clinical guidelines were implemented in Denmark. Our results indicate that new guidelines might reduce inequality in $\mathrm{OAC}$ initiation and that new, high-cost drugs increase inequality.

\section{INTRODUCTION}

Atrial fibrillation (AF) is the most common cardiac arrhythmia of clinical importance and an increasing clinical burden associated with increased mortality and morbidity, especially

\section{Strengths and limitations of this study}

- This is a large nationwide study based on all patients with incident atrial fibrillation registered at hospitals in Denmark with high risk of stroke $(\mathrm{N}=154$ 448).

- The study examines the socioeconomic trends in oral anticoagulation treatment for patients with atrial fibrillation over a long time period (1999-2016).

- The study uses individual-level socioeconomic factors.

- The study evaluates and indicates potential causes of socioeconomic differences; however, it is of observational design and true causality cannot be determined.

- Although Danish nationwide registers in general are of high quality, some limitations might occur and many clinical observations are not available in the registers.

related to heart failure and stroke. ${ }^{1}$ Oral anticoagulation (OAC) therapy is important for patients with $\mathrm{AF}$ as it prevents stroke, and current European guidelines recommend OAC to majority of patients with AF, except for those with very low risk of stroke. ${ }^{2}$ Despite these recommendations, OAC still tends to be underused in patients with AF and high risk of stroke. A systematic review from 2010 found that 25 out of 29 studies with patients with prior stroke or transient ischaemic attack showed that these patients were undertreated (defined as treatment level below $70 \%$ of the population). ${ }^{3}$ The factors that influence this are not fully known, but socioeconomic factors (SEFs) may be of importance.

Previous literature on SEFs and OAC therapy for stroke prevention in patients with AF shows varying results, ${ }^{4}$ and no study has, to our knowledge, examined temporal 
changes in treatment inequalities and the impact of clinical guidelines.

The aim of this study was to examine the association between different levels of SEFs and the initiation of OAC therapy according to guidelines in a population of Danish patients with AF between 1999 and 2016. We hypothesised that patients with AF living alone and with low income and low education received less guideline-recommended OAC therapy for AF and that the socioeconomic differences have been reduced when new guidelines were published throughout the years.

\section{METHODS}

\section{Data source}

This is a register-based nationwide study using different registers accessed through Statistics Denmark. ${ }^{5}$ The Danish National Patient Register contains information about diagnoses, which are coded using the Eighth International Classification of Diseases (ICD-8) until 1993 and the ICD-10 from 1994 and hereafter. ${ }^{67}$ The National Prescription Registry holds information on drugs dispensed, which are identified by the Anatomical Therapeutic Chemical (ATC) classification system code. ${ }^{8}$ Danish registers on personal income and transfer payments keep information about the Danish populations' income, ${ }^{9}$ and the Danish education registers hold information about educational status. ${ }^{10}$ All Danish residents have a personal identification number ('CPR-number') which allows linkage between all the registers. ${ }^{11}$

\section{Study population}

All Danish residents aged $\geq 30$ years with an incident hospital diagnosis of AF or atrial flutter between 1 May 1999 and 2 October 2015 were identified in the Danish National Patient Register as ICD-10 code I48 (AF or atrial flutter) and included in this study. The study started in 1999 because, to our knowledge, the first official European Society of Cardiology guideline was published in 1998. ${ }^{12}$ We excluded all patients with low and moderate risk of stroke as there is no definite indication for OAC in these groups. The study population of patients with high risk of stroke was determined based on the European recommendations/guidelines the respective year a patient was included, more specifically in $1998,{ }^{12} 2001,{ }^{13}$ $2006,{ }^{14} 2010^{15}$ and $2012 .{ }^{16}$ For example, in 2012, low risk of stroke was defined as $\mathrm{CHA}_{2} \mathrm{DS}_{2}$-VASc (Congestive heart failure/left ventricular dysfunction, Hypertension, Age $\geq 75$ years old [2 points], Diabetes mellitus, Stroke/ transient ischemic attack/thromboembolism [2 points], Vascular disease, Age 65 to 75 years old, Sex category [female sex 1 point] $)^{17}$ of 0 for men and 1 for women, moderate risk of stroke was defined as $\mathrm{CHA}_{2} \mathrm{DS}_{2}$-VASc 1 for men (whereas women could not have moderate risk of stroke), and high risk of stroke was $\mathrm{CHA}_{2} \mathrm{DS}_{2}-\mathrm{VASc} \geq 2$ for men and women. ${ }^{16}$ In this study, guidelines were considered to be updated by 1 May the year following publication due to a time delay from publication of European guidelines to implementation in Denmark. Each time a guideline was updated, the definition or risk factors and recommended treatments were changed. Further explanation and the specific classification of risk of stroke for each year are available in online supplemental material S1. Furthermore, we excluded all patients with missing information on income, cohabiting status and place of residence or patients with valvular AF.

\section{Income, education and cohabiting status}

The SEFs used as exposure in this study were income, level of education and cohabiting status measured at baseline. We used data on the equalised household income, ${ }^{18}$ and to account for changes in salaries over time and age we split it into age-specific (5-year intervals) and entry year-specific (every second year) tertiles. If income was missing in the year of inclusion, income from the previous year was used. We used the distribution of income in the general population of Danish residents ( $\geq 30$ years) (and not only the AF population of this study) when defining the income tertiles.

The division of education was split into date-of-birth cohorts of those born before $\leq 1965$ and after $\geq 1966$ as access to education has changed with birth cohorts. ${ }^{19}$ The year 1965 was chosen as cut-off date based on the distribution of educational status in the whole population. We divided education into low (no registered education, primary education, lower secondary education), medium (upper secondary education programmers and short-cycle tertiary education for those born $\leq 1965$, and for those born $\geq 1966$ a bachelor's degree or similar was also considered as medium education) and high (for those born $\leq 1965$, bachelor's degree, master's degree and doctor's degree were considered as high education, and for those born $\geq 1966$ only master's degree and doctor's degree were considered as high education). For further details on education, please see online supplemental material S2. Cohabiting status was divided into living 'not alone' or 'alone'.

\section{OAC therapy initiation}

Initiation of OAC was defined as at least one prescription of vitamin $\mathrm{K}$ antagonist (VKA) or novel oral anticoagulant (NOAC) within -30 to +90 days of baseline (AF diagnosis). The time window started 30 days before the $\mathrm{AF}$ diagnosis as some patients are diagnosed by a general practitioner and therefore initiated OAC treatment at the time they are first time seen at the hospital, ${ }^{20}$ and the time frame of 90 days after was chosen as it is used in other similar studies. ${ }^{21}$

Patients who claimed a prescription of both NOAC and VKA on the same day after baseline or both within baseline were counted as being initiated with NOAC. No other anticoagulation regimens other than VKA and NOACs were investigated in this study. The specific ATC codes used are available in online supplemental material S3. 


\section{Covariates}

Place of residence was classified into urban, intermediate, rural and peripheral municipalities ${ }^{22}$ as previously described. ${ }^{23}$ The same classification was used the whole study period. Information on comorbidities and medication was divided into present or not present based on ICD-8/10, ATC and surgical/procedure codes (the specific ICD and ATC codes are available in online supplemental material S3), and included heart failure, hypertension, diabetes mellitus (DM), ischaemic stroke/ transient ischaemic attack (TIA)/systemic embolism (SE), myocardial infarction (MI), ischemic heart disease (IHD), thyrotoxicosis, peripheral artery disease (PAD)/ aortic plaque, renal disease, liver disease, bleeding, alcoholism, non-steroidal anti-inflammatory drug (NSAID), antiplatelet drugs, venous thromboembolism (VTE), and hip and knee arthroplasty. Risk of stroke and risk scores such has $\mathrm{CHA}_{2} \mathrm{DS}_{2}$-VASc and HAS-BLED (Hypertension, Abnormal renal/liver function, Stroke, Bleeding, Labile INR [international normalized ratio], Elderly, Drugs/ alcohol) ${ }^{24}$ score were defined according to guidelines/ recommendations the specific year they were included ${ }^{12-16}$ (online supplemental materials S1 and S3).

\section{Statistics}

Baseline characteristics of participants were presented by mean and SD for continuous variables, and for categorical variables we used counts and percentages (\%). Normal distribution of continuous variables was checked with histograms and Q-Q plots. Figures were constructed showing OAC initiation over time according to education, income and cohabiting status. The crude figures were constructed with counting, whereas the age-adjusted curves show the predicted percentages of initiation of OAC for a 78-year-old patient, calculated from a model with interaction between enter year, exposure and age. Age 78 was chosen as this is the mean age of the participants. A generalised linear regression model was used to estimate the absolute risk of being initiated with OAC. We chose to use risk differences (RDs) as the measure of association when comparing different socioeconomic groups (eg, living alone vs not living alone). We supplied with robust standard errors to accommodate binary, nonnormal distributions of response variables.

The analyses included an unadjusted and three multivariable models. Model 1 was adjusted for age, which enters the model as a restricted cubic spline with three knots; model 2 included model 1 and other sociodemographic factors (education, income, cohabiting status and place of residence); and model 3 included model 2 and confounding comorbidity and medication, which varied with SEF and period. For income and cohabiting status, this included in 1998-2001 heart failure, hypertension, DM, ischaemic stroke/TIA/SE, MI, antiplatelet drugs, VTE, and hip and knee arthroplasty; in 2001-2006, heart failure, hypertension, DM, ischaemic stroke/TIA/ SE, MI, IHD, thyrotoxicosis, antiplatelet drugs, VTE, and hip and knee arthroplasty; in 2006-2010, heart failure, hypertension, DM, ischaemic stroke/TIA/SE, MI, IHD, thyrotoxicosis, antiplatelet drugs, VTE, and hip and knee arthroplasty; and in 2010-2012 and 2012-2016, heart failure, hypertension, DM, ischaemic stroke/TIA/SE, MI, $\mathrm{PAD} /$ aortic plaque, renal disease, liver disease, bleeding, alcoholism, NSAID, antiplatelet drugs, VTE, and hip and knee arthroplasty. For education, model 3 included model 1 and model 2, and in 1998-2001 DM and VTE; in 2001-2006, DM, thyrotoxicosis and VTE; in 2006-2010, DM, thyrotoxicosis and VTE; and in 2010-2012 and 2012-2016, DM, alcoholism, NSAID and VTE. The rationale behind the different models are available in online supplemental material $\mathrm{S} 4$. We stratified the main results on sex to account for potential effect modification.

We performed sensitivity analyses by excluding patients without any registered education and another analysis where the baseline was changes from date of admission to date of discharge for AF diagnosis. We used STATA V.16.1 for statistical analysis.

\section{Patient and public involvement statement}

No patients were involved in this study.

\section{RESULTS}

A total of 258086 patients were registered with an incident hospital diagnosis of AF between 1 May 1999 and 2 October 2015. Of these, 36876 and 59530 patients were excluded due to low and medium risk of stroke, respectively, as they had no definite indication for OAC. Furthermore, 2409 patients were excluded due to valvular AF and 4823 patients were excluded due to missing variables about exposure or covariates. In total, 154448 patients with non-valvular AF and high risk of stroke were included in the final analysis. Online supplemental figure S5 shows the identification of the study population in a flow chart.

Overall, $74551(48.3 \%)$ patients were initiated with OAC, including $56428(36.5 \%)$ with VKA and 18123 $(11.7 \%)$ with NOAC, of whom $48(0.0 \%)$ claimed a prescription of VKA the same day or both within baseline. Of the patients, 18899 (12.2\%) died during follow-up. The use of OAC increased over time; $34.2 \%$ claimed a prescription within $-30 /+90$ days after the AF diagnoses in 1999-2002, contrary to $40.1 \%, 41.2 \%, 54.5 \%$ and 67.7\% in 2002-2007, 2007-2011, 2011-2013 and 20132016, respectively.

\section{Baseline characteristics}

Table 1 shows the baseline characteristics. The mean age was 78.2 years and $47.3 \%$ of the study population were men. Low education was more common than high education. Heart failure, ischaemic stroke, MI and ischaemic heart disease were more common in the earliest years, while hypertension was the most common condition overall. We performed sensitivity analyses where we excluded patients not registered as not having any educational status, which included 30540 patients who were, in general, older (mean age 87.9), mainly women $(65 \%$ 
Table 1 Baseline characteristics according to year cohort included for patients with atrial fibrillation

\begin{tabular}{|c|c|c|c|c|c|c|}
\hline Period ${ }^{*}$ & 1999-2002 & 2002-2007 & $2007-2011$ & $2011-2013$ & 2013-2016 & All \\
\hline All & 16072 & 51767 & 25638 & 26807 & 34164 & 154448 \\
\hline Men (\%) & 7961 (49.5) & $24884(48.1)$ & 11938 (46.6) & $12172(45.4)$ & 16057 (47.0) & 73012 (47.3) \\
\hline \multicolumn{7}{|l|}{ Cases } \\
\hline OAC, n (\%) & $5493(34.2)$ & $20750(40.1)$ & $10558(41.2)$ & 14609 (54.5) & $23141(67.7)$ & 74551 (48.3) \\
\hline NOAC, n (\%) & $0(0.0)$ & $0(0.0)$ & $18(0.1)$ & 4521 (16.9) & $13584(39.8)$ & $18123(11.7)$ \\
\hline Low & $12012(74.7)$ & $35910(69.4)$ & $16227(63.3)$ & $14650(54.6)$ & $17272(50.6)$ & 96071 (62.2) \\
\hline Medium & 3077 (19.1) & $11881(23.0)$ & $7105(27.7)$ & $8880(33.1)$ & $12195(35.7)$ & $43138(27.9)$ \\
\hline High & $983(6.1)$ & $3976(7.7)$ & $2306(9.0)$ & 3277 (12.2) & $4697(13.7)$ & $15239(9.9)$ \\
\hline \multicolumn{7}{|l|}{ Income tertiles, $\mathrm{n}(\%) \ddagger$} \\
\hline Low & $5458(34.0)$ & $17665(34.1)$ & 9198 (35.9) & 9415 (35.1) & $12344(36.1)$ & $54080(35.0)$ \\
\hline Peripheral & $1635(10.2)$ & $5446(10.5)$ & $2723(10.6)$ & $2861(10.7)$ & 3606 (10.6) & 16271 (10.5) \\
\hline Rural & $4562(28.4)$ & $15401(29.8)$ & 7502 (29.3) & $8070(30.1)$ & 10415 (30.5) & $45950(29.8)$ \\
\hline Intermediate & 2495 (15.5) & $7963(15.4)$ & 4066 (15.9) & $4433(16.5)$ & $5530(16.2)$ & 24487 (15.9) \\
\hline Urban & $7380(45.9)$ & $22957(44.3)$ & $11347(44.3)$ & $11443(42.7)$ & $14613(42.8)$ & 67740 (43.9) \\
\hline \multicolumn{7}{|l|}{ Cohabiting status, n (\%) } \\
\hline Alone & $6930(43.1)$ & $23127(44.7)$ & $11927(46.5)$ & $11514(43.0)$ & $14589(42.7)$ & $68087(44.1)$ \\
\hline Not alone & $9142(56.9)$ & $28640(55.3)$ & $13711(53.5)$ & $15293(57.0)$ & $19575(57.3)$ & 86361 (55.9) \\
\hline \multicolumn{7}{|l|}{ Comorbidity, n (\%) } \\
\hline Heart failure & 3099 (19.3) & $5587(10.8)$ & 3997 (15.6) & $2127(7.9)$ & $2456(7.2)$ & $17266(11.2)$ \\
\hline PAD or aortic plaque & $1091(6.8)$ & $3055(5.9)$ & $2073(8.1)$ & $1712(6.4)$ & $2235(6.5)$ & $10166(6.6)$ \\
\hline Abnormal renal function & $534(3.3)$ & $1717(3.3)$ & $1379(5.4)$ & $1261(4.7)$ & $1865(5.5)$ & $6756(4.4)$ \\
\hline Abnormal liver function & $<10$ & $<10$ & $<10$ & $<10$ & $<10$ & $<10$ \\
\hline Bleeding & 1969 (12.3) & $7074(13.7)$ & $4250(16.6)$ & $3851(14.4)$ & 4995 (14.6) & $22139(14.3)$ \\
\hline Alcoholism & $283(1.8)$ & $1128(2.2)$ & $678(2.6)$ & $714(2.7)$ & $1082(3.2)$ & $3885(2.5)$ \\
\hline Hyperthyroidism & $574(3.6)$ & $2086(4.0)$ & $1016(4.0)$ & $853(3.2)$ & $1074(3.1)$ & $5603(3.6)$ \\
\hline Venous thromboembolism & $120(0.7)$ & $367(0.7)$ & $277(1.1)$ & $301(1.1)$ & $383(1.1)$ & $1448(0.9)$ \\
\hline Knee or hip arthroplasty surgery & $43(0.3)$ & $208(0.4)$ & $131(0.5)$ & $150(0.6)$ & $166(0.5)$ & $698(0.5)$ \\
\hline \multicolumn{7}{|l|}{ Concomitant medication } \\
\hline Antiplatelet & $8527(53.1)$ & $26098(50.4)$ & $16603(64.8)$ & $13826(51.6)$ & $16154(47.3)$ & $81208(52.6)$ \\
\hline NSAID & $4493(28.0)$ & $15732(30.4)$ & $7259(28.3)$ & $7062(26.3)$ & $7417(21.7)$ & $41963(27.2)$ \\
\hline Mean $\mathrm{CHA}_{2} \mathrm{DS}_{2}$-VASc (SD) & $3.9(1.2)$ & $3.3(1.3)$ & $4.2(1.1)$ & $3.5(1.3)$ & $3.4(1.2)$ & $3.6(1.3)$ \\
\hline Mean HAS-BLED (SD) & $2.6(0.9)$ & $2.4(1.0)$ & $3.0(0.9)$ & $2.6(1.0)$ & $2.5(1.0)$ & $2.6(1.0)$ \\
\hline
\end{tabular}

${ }^{*}$ The periods are from 1 May the following year a European guideline was published as there is normally some time from publication to implementation. For example, we used the guideline published in 1998 from 1 May 1999. Please see online supplemental material S1 for further details.

†Education is divided into low: $\leq$ lower secondary education; medium: $\leq$ short-cycle tertiary education (if birth year $\leq 1965$ ) or $\leq$ bachelor's degree or similar (if birth year $>1965$ ); high: zbachelor's degree or similar (if birth year $\leq 1965$ ) or $\geq$ master's degree or similar (if birth year $>1965$ ).

†lncome is divided into age-specific (5-year intervals) and enter year-specific (every second year) tertiles.

$\mathrm{CHA}_{2} \mathrm{DS}_{2}$-VASc, Congestive heart failure/left ventricular dysfunction, Hypertension, Age $\geq 75$ years old (2 points), Diabetes mellitus, Stroke/transient ischemic attack/thromboembolism (2 points), Vascular disease, Age 65 to 75 years old, Sex category (female sex 1 point); HAS-BLED, Hypertension, Abnormal renal/liver function, Stroke, Bleeding, Labile INR

(international normalized ratio), Elderly, Drugs/alcohol; NOAC, novel oral anticoagulant; NSAID, non-steroidal anti-inflammatory drug; OAC, oral anticoagulant; PAD, peripheral artery diseases. 

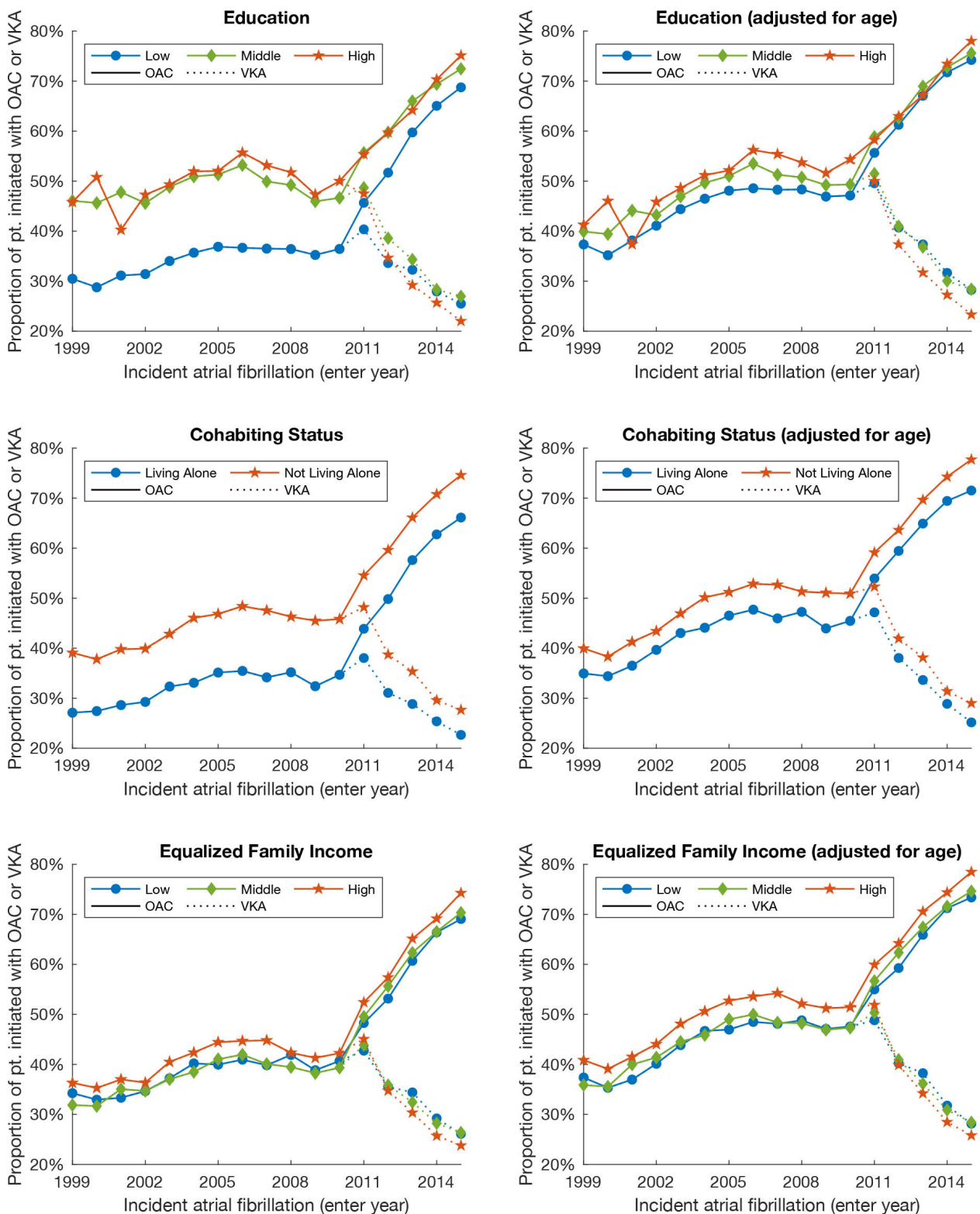

Figure 1 Proportion of patients with AF and high risk of stroke (which means they should have received OAC according to guidelines of the specific year of inclusion) being initiated with OAC (VKA or NOAC) or VKA alone with different levels of SEFs. To the right is the same age-adjusted graph for patients with AF 78 years of age. AF, atrial fibrillation; NOAC, novel oral anticoagulant; OAC, oral anticoagulation; pt., patients; SEFs, socioeconomic factors; VKA, vitamin K antagonist.

vs $35 \%$ ) and living alone (60\% vs $40 \%$ ) (online supplemental material S6, table S6a).

\section{Initiation of OAC according to SEF}

OAC initiation trends over time stratified by SEF

Figure 1 shows the percentage of patients with high risk of stroke (which means they should have received OAC according to guidelines of the specific year of inclusion) who are being initiated with OAC. It shows that initiation of OAC substantially increased over time, and that patients with low education, low income and living alone received less OAC in the form of VKA from 1999 to 2011. However, this trend changed by introduction of NOAC in 2011, and patients with low education and low income received more or roughly the same treatment with VKA, but the difference in OAC initiation between different levels education and cohabiting status seemed to be reduced around 2010-2011. The age-adjusted average trend of OAC initiation for an average patient of 78 years of age is shown in the right frame, indicating a smaller difference.

Education and initiation of OAC stratified by guideline period For men and women, patients with medium and high education received more OACs compared with patients with low education, and this inequality reduced with time. For example, the crude RD (95\% CI) in 2002-2007 for women with high education (low education as a reference) was $14.3 \%$ (11.6 to 17.0), while in 2013-2016 this reduced to $4.5 \%$ (2.4 to 6.7 ). After adjusting for age, the 
corresponding difference in OAC initiation in 2002-2007 reduced to $3.3 \%$ (0.6 to 6.0 ) and in 2013-2016 this was $0.8 \%(-1.4$ to 3.0$)$. The same trend was seen for men and for NOAC; however, crude inequality increased from $5.7 \%$ (3.6 to 7.8 ) in $2011-2013$ to $9.1 \%$ (6.9 to 11.4 ) in 2013-2016 for men and the same trend was seen for women (tables 2 and 3 ).

\section{Income and initiation of OAC stratified by guideline period}

Men with low income received less OAC compared with men with high income, but this trend was not substantially changed with time or after adjusting for potential confounders. For example, the crude RD (95\% CI) for men with the highest versus lowest income was $4.7 \%$ (2.1 to 7.3) in 1999-2002 and 5.8\% (4.1. to 7.6) in 2013-2016, and after adjusting for all potential confounders $3.9 \%$ (1.2 to 6.6 ) and $4.5 \%$ (2.6 to 6.4), respectively. For women, no strong association between income and OAC was seen; for example, the crude $\mathrm{RD}$ for women with the highest income was $-1.3 \%$ ( -3.9 to 1.2$)$ in $1999-2002$ and $1.7 \%$ ( -0.0 to $3.4)$ in 2013-2016. For both men and women, there was a substantial inequality for patients with low versus high income in initiation of NOAC, and this inequality increased with time (eg, crude RD for men with high income was $5.0 \%$ (3.3 to 6.6) in 2011-2013 and increased to $9.4 \%$ (7.6 to 11.2) in 2013-2016) (tables 2 and 3).

\section{Cohabiting status and initiation of OAC stratified by guideline period}

Men and women living alone were less likely to receive OAC and this trend slightly decreased from 2002-2007 to 2013-2016. For example, the crude RD (95\% CI) for men living alone versus not living alone in 2002-2007 and 2013-2016 was $10.2 \%$ (8.8 to 11.5 ) and $7.9 \%$ (6.3 to 9.4), respectively. After adjusting for age, the RD (95\% CI) in 2002-2006 and 2013-2016 was 6.0\% (4.7 to 7.3) and $6.3 \%$ (4.8 to 7.9). A similar trend was seen for women. For NOAC, there was a small inequality which slightly increased with time for both men and women. In 20112013, the crude RD (95\% CI) for women was $1.5 \%$ ( 0.3 to 2.7 ) and in 2013-2016 was $4.1 \%$ (2.6 to 5.5), and for men $1.1 \%$ ( -0.3 to 2.5 ) and $4.1 \%$ (2.5 to 5.7 ), respectively. The results were attenuated after adjusting for confounding variables (tables 2 and 3 ).

\section{Sensitivity analyses}

Sensitivity analyses showed that after excluding those with no education registered, the same trend between SEFs and OAC was observed, but the magnitude of the association was reduced and the difference was more constant or increased (online supplemental material S6). When using date of discharge as baseline instead of date of admission, no change of any importance was seen in the results (online supplemental material $\mathrm{S} 7$ ).

\section{DISCUSSION}

In this study we examined the association between different levels of SEFs and the initiation of OAC therapy according to guidelines in the period 1999-2016, and we found that (1) patients with high income, high education and not living alone were more often initiated with guideline-recommended OAC treatment compared with patients with low income, low education and living alone; (2) guideline-recommended initiation of OAC therapy substantially increased over time and there was a decrease in the unadjusted educational inequality; and (3) the observed inequality was strongly attenuated after adjusting for age, indicating that age is an important confounding variable in the relationship between SEFs and OAC treatment. After adjusting for other sociodemographic factors and comorbidity as well, the association was reduced even more. (4) Patients with low income, low education and living alone compared with patient with high income, high education and not living alone received less NOAC and this inequality increased with time.

\section{Interpretation of the results}

Previous research on SEFs and OAC treatment for patients with $\mathrm{AF}$ has shown varying results as several studies found no significant association. ${ }^{25-28}$ However, the mentioned studies do not seem to have excluded patients with low and moderate risk of stroke, which may have attenuated the association as patients with low education and high deprivation in general have more morbidity, ${ }^{29}$ and consequently probably a higher stroke risk score and therefore a more definite indication for OAC initiation. Furthermore, the studies did not examine the association over a long time, and they were of relatively small sample sizes, making them less comparable with our study.

We observed that low level of income, low level of education and living alone were associated with a lower chance of being initiated with NOAC and that the inequality in NOAC initiation increased with time. Even though drugs are partly reimbursed in Denmark, NOACs are still more expensive compared with VKA, and this seems to be an obvious cause of why NOACs are more often used by patients with high income, high education and not living alone. This explanation is strengthened by the fact that high income was a strong SEF for patients using NOAC. However, it does not explain the fact that high-risk patients with $\mathrm{AF}$ with low level of education, low income and living alone seemed to be undertreated with OAC (NOAC or VKA), as VKA is cheap and should be an alternative for everyone. As shown in figure 2, several factors might have a role in the socioeconomic inequality in OAC initiation. Physicians' clinical judgement is known to play an important role in OAC prescribing, and some typical causes given for not initiating OAC drugs include poor compliance, high bleeding risk and older age. ${ }^{30}$ It is paradoxical that OAC often is detained from older patients as older age is one of the strongest risk factors for stroke $^{2}$; however, it might explain the large educational inequality observed in our results as it was strongly attenuated after adjusting for age. Notwithstanding, one might argue that age itself is a social variable as the social position to some degree changes as one gets older. Also, it is 
Table 2 Risk difference and 95\% Cl of initiation of OAC (NOAC or VKA) or NOAC according to SEFs in year cohorts for women

Proportion OAC, low SEP (\%) Crude

Model 1: age

Model 2: age and sociodemographic

Model 3: age, sociodemographic and comorbidity

OAC

Education

1999-2002 26.9 (25.9 to 28.0$)$

Ref: low

Ref: low

Ref: low

Ref: low

Medium

13.8 (10.4 to 17.1)

$2.1(-1.5$ to 5.7$)$

$2.3(-1.3$ to 5.9$)$

$2.1(-1.5$ to 5.7$)$

High

11.1 (5.3 to 16.9)

0.4 ( -5.5 to 6.4$)$

$-0.3(-6.3$ to 5.8$)$

$-0.5(-6.5$ to 5.6$)$

2002-2007

31.9 (31.2 to 32.5$)$

Ref: low

Ref: low

Ref: low

Medium

14.0 (12.4 to 15.7)

2.7 (0.9 to 4.4 )

2.9 (1.1 to 4.6$)$

2.6 (0.8 to 4.4 )

High

2007-201

33.6 (32.7 to 34.5$)$

14.3 (11.6 to 17.0 )

3.3 (0.6 to 6.0)

2.5 (-0.3 to 5.4)

2.4 (-0.5 to 5.2)

Medium

Ref: low

Ref: low

Ref: low

10.2 (8.1 to 12.3 )

$1.9(-0.3$ to 4.0$)$

2.5 (0.3 to 4.7)

Ref: low

High

2011-2013

47.8 (46.8 to 48.9 )

12.3 (9.0 to 15.7 )

4.9 (1.5 to 8.3 )

4.3 (0.8 to 7.8 )

2.4 (0.2 to 4.6$)$

Medium

Ref: low

Ref: low

Ref: low

4.1 (0.6 to 7.6 )

High

8.5 (6.6 to 10.3)

2.1 (0.2 to 4.0$)$

2.5 (0.6 to 4.5$)$

Ref: low

7.1 (4.4 to 9.8 )

0.6 (-2.1 to 3.3)

-0.2 (-3.1 to 2.7$)$

2.4 (0.4 to 4.3 )

2013-2016

64.3 (63.4 to 65.2) Ref: low

Medium

3.6 (2.1 to 5.2$)$

Ref: low

Ref: low

-0.4 ( -3.3 to 2.5$)$

High

4.5 (2.4 to 6.7$)$

$0.5(-1.0$ to 2.1$)$

$0.9(-0.7$ to 2.5$)$

Ref: low

Income

\begin{tabular}{|c|c|c|c|c|c|}
\hline 1999-2002 & 30.3 (28.7 to 32.0 ) & Ref: low & Ref: low & Ref: low & Ref: low \\
\hline Medium & & $-3.0(-5.3$ to -0.6$)$ & $-1.0(-3.2$ to 1.3$)$ & $-0.7(-3.0$ to 1.5$)$ & $-0.9(-3.2$ to 1.3$)$ \\
\hline High & & $-1.3(-3.9$ to 1.2$)$ & $1.3(-1.1$ to 3.7$)$ & $1.7(-0.8$ to 4.2$)$ & $1.2(-1.3$ to 3.7$)$ \\
\hline Medium & & $-0.3(-1.7$ to 1.0$)$ & 0.9 (-0.4 to 2.2$)$ & $1.1(-0.2$ to 2.4$)$ & 1.0 (-0.3 to 2.3$)$ \\
\hline High & & $0.2(-1.2$ to 1.7$)$ & 2.7 (1.3 to 4.1$)$ & 3.0 (1.6 to 4.4$)$ & 2.5 (1.1 to 4.0$)$ \\
\hline $2007-2011$ & 37.5 (36.1 to 38.8$)$ & Ref: low & Ref: low & Ref: low & Ref: low \\
\hline High & & $-0.4(-2.4$ to 1.6$)$ & 2.2 (0.3 to 4.2$)$ & $2.6(0.5$ to 4.7$)$ & $2.2(0.1$ to 4.3$)$ \\
\hline 2011-2013 & 51.2 (49.9 to 52.5 ) & Ref: low & Ref: low & Ref: low & Ref: low \\
\hline Medium & & $-1.6(-3.5$ to 0.3$)$ & $-0.4(-2.2$ to 1.5$)$ & $0.2(-1.6$ to 2.1$)$ & $0.1(-1.8$ to 1.9$)$ \\
\hline High & & $0.5(-1.5$ to 2.5$)$ & $2.0(-0.0$ to 3.9$)$ & 3.4 (1.2 to 5.5$)$ & $3.2(1.0$ to 5.3$)$ \\
\hline \multicolumn{6}{|c|}{ Cohabiting status } \\
\hline 1999-2002 & 25.1 (23.9 to 26.4 ) & Ref: low & Ref: low & Ref: low & Ref: low \\
\hline Not alone & & 8.6 (6.6 to 10.6$)$ & $2.5(0.4$ to 4.5$)$ & $1.9(-0.2$ to 3.9$)$ & $1.7(-0.3$ to 3.8$)$ \\
\hline 2002-2007 & 30.7 (30.0 to 31.4$)$ & Ref: low & Ref: low & Ref: low & Ref: low \\
\hline Not alone & & 9.5 (8.3 to 10.6$)$ & 3.0 (1.9 to 4.2$)$ & 2.4 (1.2 to 3.6$)$ & 2.4 (1.2 to 3.5$)$ \\
\hline 2007-2011 & 31.9 (30.9 to 32.9$)$ & Ref: low & Ref: low & Ref: low & Ref: low \\
\hline Not alone & & 10.8 (9.1 to 12.4$)$ & 4.7 (3.0 to 6.3 ) & 3.6 (1.9 to 5.2$)$ & 3.4 (1.7 to 5.0$)$ \\
\hline 2011-2013 & 46.4 (45.3 to 47.5$)$ & Ref: low & Ref: low & Ref: low & Ref: low \\
\hline Not alone & & 9.2 (7.6 to 10.8$)$ & 3.7 (2.0 to 5.3 ) & 2.5 (0.9 to 4.2$)$ & 2.0 (0.4 to 3.7$)$ \\
\hline
\end{tabular}

Continued 


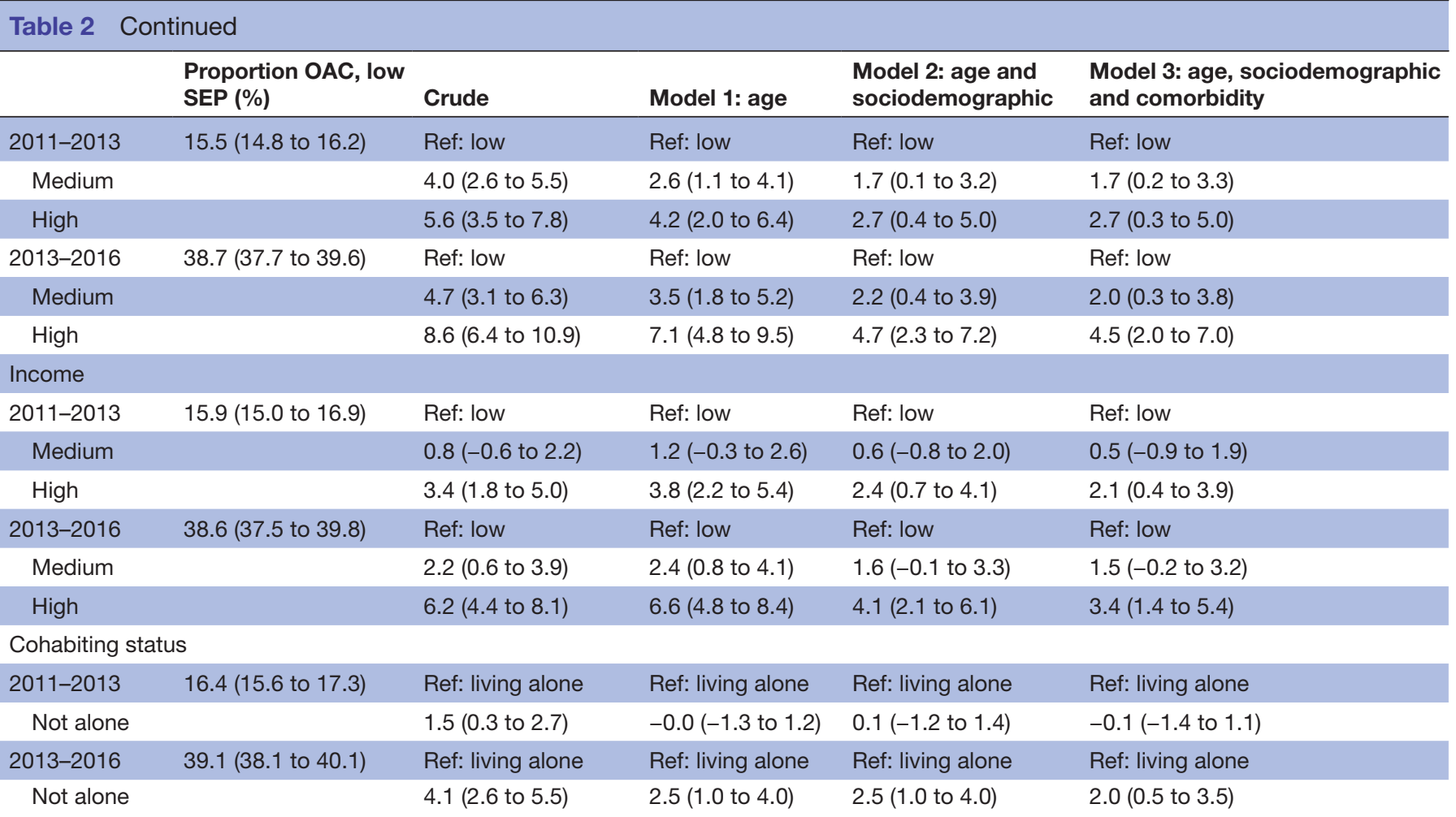

Education: crude: the unadjusted analysis. Model 1: adjusted for age. Model 2: adjusted for sociodemographic factors: model 1, household income, cohabiting status and place of residence. Model 3: adjusted for model 1, model 2 and 1998-2001: diabetes mellitus and VTE; 2001-2006: diabetes mellitus, thyrotoxicosis and VTE; 2006-2010: diabetes mellitus, thyrotoxicosis and VTE; 2010-2012: diabetes mellitus, alcoholism, NSAID and VTE; 2012-2016: diabetes mellitus, alcoholism, NSAID and VTE.

Income: crude: the unadjusted analysis. Model 1: adjusted for age. Model 2: adjusted for sociodemographic factors: model 1, education, cohabiting status and place of residence. Model 3: adjusted for model 1, model 2 and 1998-2001: heart failure, hypertension, diabetes mellitus, ischaemic stroke/TIA/systemic embolism, myocardial infarction, antiplatelet drugs, VTE, and hip and knee arthroplasty; 2001-2006: heart failure, hypertension, diabetes mellitus, ischaemic stroke/TIA/systemic embolism, myocardial infarction, ischemic heart disease, thyrotoxicosis, antiplatelet drugs, VTE, and hip and knee arthroplasty; 2006-2010: heart failure, hypertension, diabetes mellitus, ischaemic stroke/TIA/systemic embolism, myocardial infarction, ischemic heart disease, thyrotoxicosis, antiplatelet drugs, VTE, and hip and knee arthroplasty; 2010-2012: heart failure, hypertension, diabetes mellitus, ischaemic stroke/TIA/systemic embolism, myocardial infarction, peripheral artery disease/aortic plaque, renal disease, liver disease, bleeding, alcoholism, NSAID, antiplatelet drugs, VTE, and hip and knee arthroplasty; 2012-2016: heart failure, hypertension, diabetes mellitus, ischaemic stroke/TIA/systemic embolism, myocardial infarction, peripheral artery disease/aortic plaque, renal disease, liver disease, bleeding, alcoholism, NSAID, antiplatelet drugs, VTE, and hip and knee arthroplasty.

Cohabiting status: the same models as for income.

NOAC, novel oral anticoagulant; NSAID, non-steroidal anti-inflammatory drug; OAC, oral anticoagulation; Ref, reference; SEFs, socioeconomic factors; SEP, socioeconomic position; TIA, transient ischaemic attack; VKA, vitamin K antagonist; VTE, venous thromboembolism.

well known that patients with low level of education and high deprivation in general tend to have more comorbidity. ${ }^{29}$ Hence, it is possible that some of the socioeconomic inequality observed in our result is confounded or mediated through other factors simply because patients with low level of education, low income and living alone were older, sicker and had more polypharmacy. This assumption is also strengthened by the fact that the association was strongly attenuated after adjusting for age, comorbidity and other drugs (tables 2 and 3 ).

Also, patients with low education and low income tend to have low health literacy ${ }^{31}$ and potentially poor compliance, and it is possible that the physician has prescribed an OAC, but that the patient never claimed it (our data only register fulfilled prescriptions) because they, for example, did not understand the importance of OAC for stroke prevention for patients with AF and high risk of stroke. More focus on patient information and education could be a target for public health authorities in order to avoid this potential issue.

Our results also showed that the overall OAC use has improved over time and that VKA was replaced by NOAC as the most prescribed OAC, which is also consistent with previous studies. ${ }^{20} 32$

However, we also found that the unadjusted socioeconomic inequality in OAC treatment reduced over time, which, to our knowledge, has not been shown before. However, a similar trend has been seen in other diseases; for example, a Danish study found increased use of coronary angiography (CAG) on day 1 and day 3 for patients with NSTEMI (non-ST-segment elevation myocardial infarction) across all educational groups along with reduced inequality. They suggested that implementation of clinical guidelines ensured more uniform treatment for all patients with NSTEMI, and that this, among other things, may have decreased the educational inequality they found in access 
Table 3 Risk difference and $95 \% \%$ Cl of initiation of OAC (NOAC or VKA) or NOAC according to SEFs in year cohorts for men

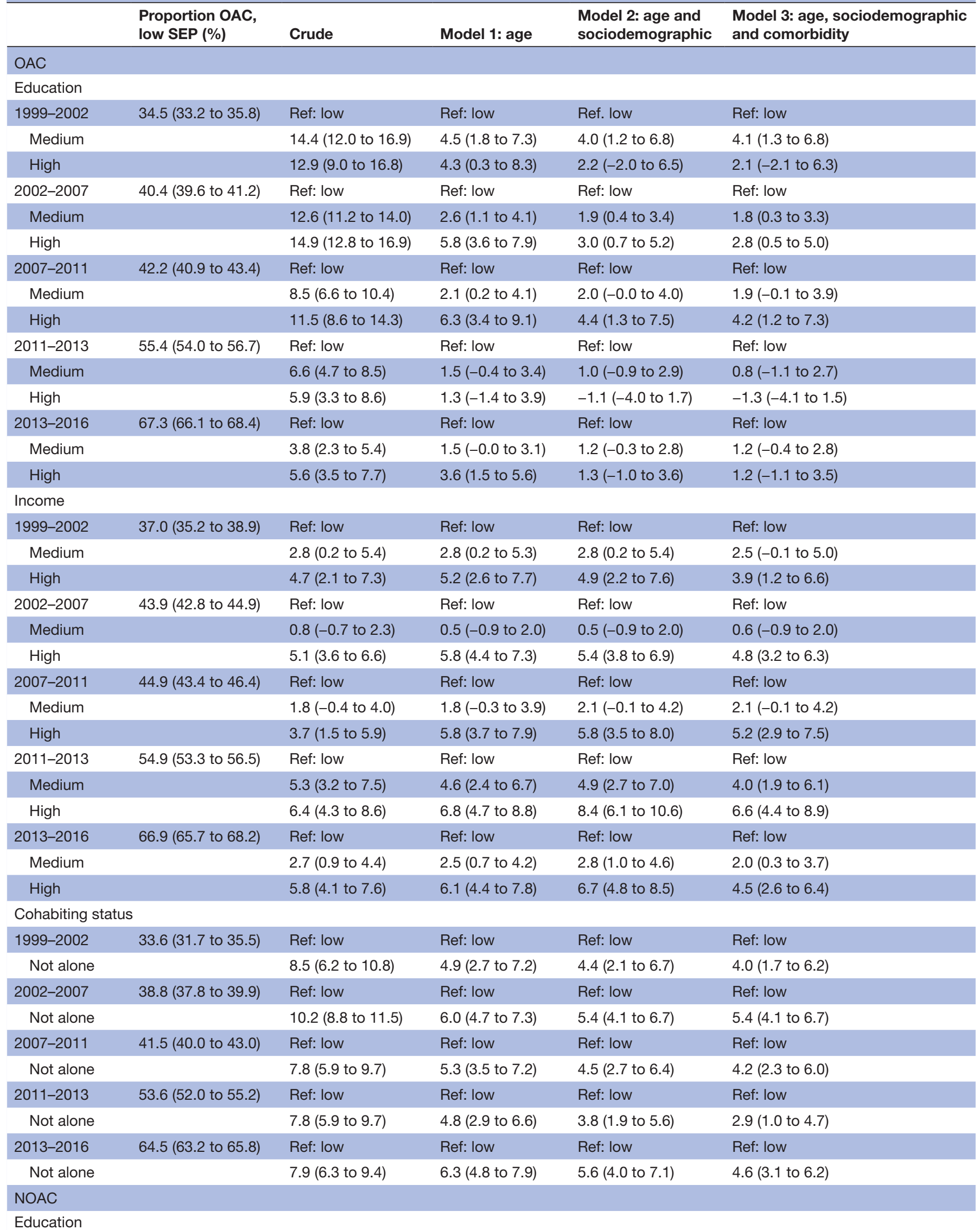




\begin{tabular}{|c|c|c|c|c|c|}
\hline & $\begin{array}{l}\text { Proportion OAC, } \\
\text { low SEP (\%) }\end{array}$ & Crude & Model 1: age & $\begin{array}{l}\text { Model 2: age and } \\
\text { sociodemographic }\end{array}$ & $\begin{array}{l}\text { Model 3: age, sociodemographic } \\
\text { and comorbidity }\end{array}$ \\
\hline 2011-2013 & 14.6 (13.6 to 15.5$)$ & Ref: low & Ref: low & Ref: low & Ref: low \\
\hline Medium & & 2.8 (1.4 to 4.2$)$ & $1.7(0.2$ to 3.1$)$ & $0.8(-0.7$ to 2.2$)$ & $0.6(-0.8$ to 2.1$)$ \\
\hline High & & 5.7 (3.6 to 7.8 ) & 4.7 (2.6 to 6.9 ) & $2.0(-0.3$ to 4.3$)$ & $1.9(-0.4$ to 4.1$)$ \\
\hline 2013-2016 & 35.3 (34.2 to 36.5$)$ & Ref: low & Ref: low & Ref: low & Ref: low \\
\hline Medium & & 3.5 (1.9 to 5.1$)$ & 2.4 (0.8 to 4.1$)$ & $0.9(-0.7$ to 2.6$)$ & $0.9(-0.8$ to 2.5$)$ \\
\hline High & & 9.1 (6.9 to 11.4$)$ & 8.2 (5.9 to 10.5$)$ & 3.3 (0.8 to 5.8 ) & 3.2 (0.7 to 5.7 ) \\
\hline \multicolumn{6}{|l|}{ Income } \\
\hline 2011-2013 & 14.4 (13.3 to 15.5$)$ & Ref: low & Ref: low & Ref: low & Ref: low \\
\hline Medium & & $1.3(-0.3$ to 2.8$)$ & $1.2(-0.4$ to 2.7$)$ & $1.0(-0.6$ to 2.6$)$ & $0.5(-1.0$ to 2.1$)$ \\
\hline High & & 5.0 (3.3 to 6.6$)$ & 5.2 (3.6 to 6.8$)$ & 4.0 (2.2 to 5.7$)$ & 3.1 (1.4 to 4.9 ) \\
\hline 2013-2016 & 34.1 (32.9 to 35.4$)$ & Ref: low & Ref: low & Ref: low & Ref: low \\
\hline Medium & & 2.8 (1.0 to 4.7 ) & 2.8 (1.0 to 4.6$)$ & 2.3 (0.5 to 4.2$)$ & 1.8 (0.0 to 3.6$)$ \\
\hline High & & 9.4 (7.6 to 11.2$)$ & 9.6 (7.8 to 11.4$)$ & 7.6 (5.6 to 9.6 ) & 6.0 (3.9 to 8.0 ) \\
\hline \multicolumn{6}{|c|}{ Cohabiting status } \\
\hline 2011-2013 & $15.8(14.6$ to 16.9$)$ & Ref: living alone & Ref: living alone & Ref: living alone & Ref: living alone \\
\hline Not alone & & $1.1(-0.3$ to 2.5$)$ & $0.5(-1.0$ to 1.9$)$ & $0.2(-1.2$ to 1.6$)$ & $-0.2(-1.6$ to 1.2$)$ \\
\hline 2013-2016 & 35.5 (34.2 to 36.8$)$ & Ref: living alone & Ref: living alone & Ref: living alone & Ref: living alone \\
\hline Not alone & & 4.1 (2.5 to 5.7 ) & 3.3 (1.7 to 4.9$)$ & 2.7 (1.1 to 4.3$)$ & 2.0 (0.4 to 3.6$)$ \\
\hline
\end{tabular}

The same models as in table 2 .

NOAC, novel oral anticoagulant; OAC, oral anticoagulation; Ref, reference; SEFs, socioeconomic factors; SEP, socioeconomic position; VKA, vitamin $\mathrm{K}$ antagonist.

to $\mathrm{CAG} \cdot{ }^{33} \mathrm{~A}$ similar scenario might be the case in relation to SEFs and OAC treatment for patients with AF. The introduction of a simple and uniform stroke risk stratification score, such as the $\mathrm{CHA}_{2} \mathrm{DS}_{2}$-VASc score, might have contributed to more equal treatment for $\mathrm{AF}$ which is observed in our results.

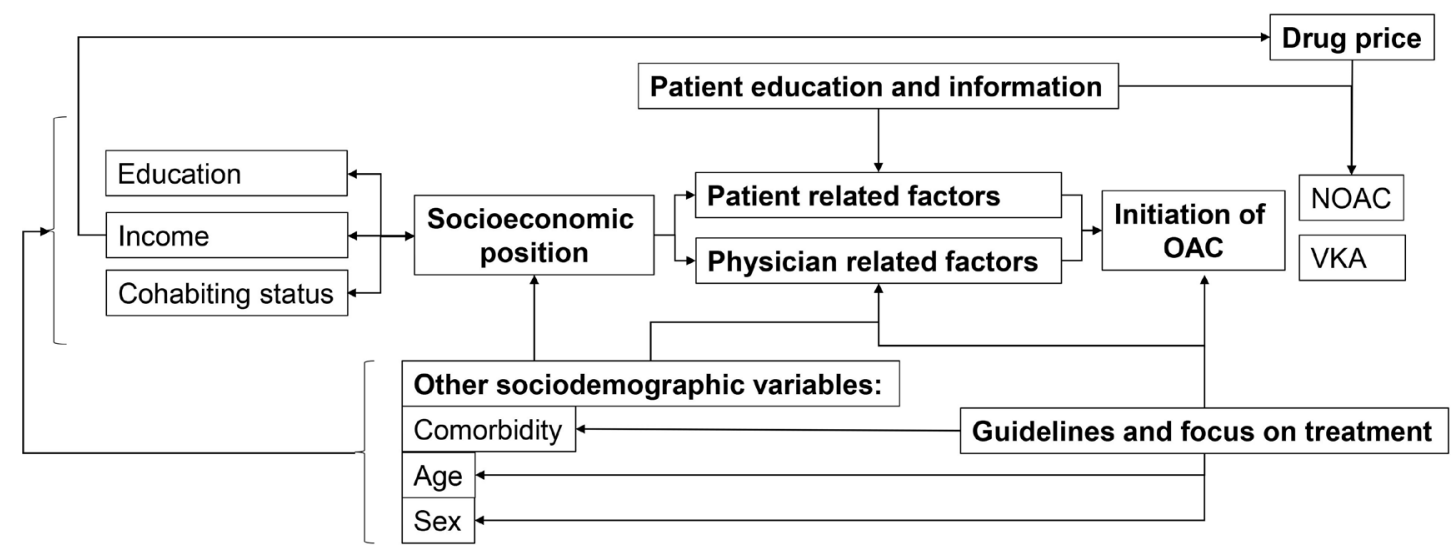

Figure 2 Hypothesised pathways and relevant variables associated with the association between SEP and OAC initiation. SEP is indicated by, for example, education, income and cohabiting status. Furthermore, other variables such as sex, age and comorbidity may also influence the SEP a person has. The intermediate step of how SEP is associated with initiation of OAC may be mediated through patient-related factors such patients' attitude towards OAC, patients who forget to dispense prescriptions or patients who do not understand why they should take OAC. Patient education and information may reduce patient-related factors limiting use of OAC. Physician-related factors include physicians' attitude towards OAC, physicians' knowledge and understanding of guidelines, physicians overestimating (or in some cases correctly estimating) bleeding risk due to, for example, older age and multimorbidity, and physicians intentionally or unintentionally discriminating patients with low SEP or factors associated with low SEP. Precise, simple and unambiguous guidelines might help reduce physicianrelated factors in the barrier to initiating OAC. Drug price may be related to low income and therefore less likelihood of being initiated with NOAC. NOAC, novel oral anticoagulant; OAC, oral anticoagulation; SEP, socioeconomic position; VKA, vitamin K antagonist. 
Systematic inequality in treatment is a concern which should be acted on and reduced in a welfare society such as Denmark.

Future research should explore the causes of socioeconomic differences in initiation of OAC, including if the reasons are patient-related (not claiming the prescription) or physician-related (not prescribing). The latter can potentially be reduced with simple and unambiguous guidelines, while the first can be reduced with, for example, patient education and information.

\section{Strengths and limitations}

This study has some limitations which should be noted when interpreting the results. First, although Danish register data in general are of good quality, a large proportion of the oldest patients are not registered with any education, possibly because they do not have any formal education, but it may also be because it is missing in the register, especially because the validity of educational status is limited for patients born before $1921 .{ }^{34}$ However, we did not exclude patients with no educational status registered in our main results as this introduces a large selection bias into our population where older, probably poorer educated and mostly women would be excluded (online supplemental table S6a). Another limitation is that it is important to notify that we cannot discriminate if the cause of not being on OAC is 'patient related' or 'physician related' factors, as we can only see prescriptions which are claimed. Hence, we do not know if the patients were not initiated because they did not claim the prescription or because the physician did not prescribe it. Another consideration is that the true risk of stroke may vary beyond what is registered in this study as there is varying validity of the diagnoses used to classify risk of stroke (online supplemental material S3). Furthermore, there may be clinical and/or patient-related factors influencing OAC initiation that are not available in the registers. One example is cases of postoperative AF where clinicians may have estimated that OAC was not beneficial for the specific patient. Lastly, even though the validity of the $\mathrm{AF}$ /atrial flutter diagnosis is good (positive predictive value found to be $93 \%^{35}$ and $99 \%^{36}$ ), it is important to note that we were only able to include hospital-registered patients with AF. Some patients with AF might be diagnosed and treated by their general practitioner and therefore never registered in the register. However, it is possible that most patients with $\mathrm{AF}$ will be referred to the hospital for, for example, direct current conversion or echocardiography. ${ }^{35}$

A great strength of this study is the large sample size and nearly complete follow-up from Danish registers. ${ }^{7}$ We have stratified the patients according to stroke risk the respective year they were included and accounted for difference in applying guidelines, used different SEFs and adjusted for a variety of potential confounders which may have influenced the result. We also accounted for almost all pitfalls when using income as an indicator by using equivalised family income to account for size of the family, enter year to account for inflation, and age to account for the difference in income for older versus younger persons. Another great strength of this study is that all the socioeconomic data we used are individuallevel data and not area-based.

\section{CONCLUSION}

Low level of income, low level of education and living alone were associated with lower chance of being initiated with OAC, and for education and cohabiting status the crude difference reduced around 2011 when more detailed clinical guidelines were implemented in Denmark. Our results indicate that specified guidelines might reduce inequality in OAC initiation and that new, high-cost drugs may increase inequality.

\section{Author affiliations}

${ }^{1}$ Department of Cardiology, Aalborg University Hospital, Aalborg, Denmark ${ }^{2}$ Aalborg AF Study Group, Aalborg University Hospital, Aalborg, Denmark ${ }^{3}$ Department of Clinical Medicine, Aalborg University, Aalborg, Denmark ${ }^{4}$ Department of Social Medicine, Aalborg University Hospital, Aalborg, Denmark

${ }^{5}$ Unit of Clinical Biostatistics, Aalborg University Hospital, Aalborg, Denmark

${ }^{6}$ Danish Center for Clinical Health Services Research, Department of Clinical Medicine, Aalborg University, Aalborg, Denmark

${ }^{7}$ Liverpool Centre for Cardiovascular Science, University of Liverpool and Liverpool Heart and Chest Hospital, Liverpool, UK

${ }^{8}$ Department of Clinical Medicine, Aalborg Thrombosis Research Unit, Aalborg University, Aalborg, Denmark

Correction notice This article has been corrected since it was published. Author name 'Gregory YH Lip' has been updated.

Acknowledgements The authors would like to thank Martin Nygård Johansen for support with data management and useful discussions with regard to the overall planning of this study.

Contributors EDL drafted the analysis plan. AMJ, SL-C, KF, SPJ, MLL and SR revised it critically and contributed to conception, design and analyses of the work. EDL, AMJ, SL-C, KF, SPJ, MLL, GYHL and SR interpreted the data for this work. EDL drafted the manuscript and AMJ, SL-C, KF, SPJ, MLL, GYHL and SR revised it critically. EDL, AMJ, SL-C, KF, SPJ, MLL, GYHL and SR gave final approval for the final version of this manuscript.

Funding This article was partly funded by the Obel Family Foundation and the Karen Elise Jensen Foundation. The funders had no role in the work or publishing of this study.

Competing interests GYHL: consultant for Bayer/Janssen, BMS/Pfizer, Boehringer Ingelheim, Verseon, Daiichi Sankyo, Biotronik, Microlife and Medtronic; speaker for Bayer, BMS/Pfizer, Boehringer Ingelheim, Daiichi Sankyo, Roche, Medtronic and Microlife. No fees are directly received personally. EDL, AMJ, SL-C, KF, SPJ, MLL and SR declare no conflict of interest.

Patient consent for publication Not required.

Ethics approval In Denmark, ethical approval is not needed for register-based studies.

Provenance and peer review Not commissioned; externally peer reviewed.

Data availability statement No data are available. According to Danish law, data sharing is not allowed because data access is only given to researchers directly responsible for conducting a specific study.

Supplemental material This content has been supplied by the author(s). It has not been vetted by BMJ Publishing Group Limited (BMJ) and may not have been peer-reviewed. Any opinions or recommendations discussed are solely those of the author(s) and are not endorsed by BMJ. BMJ disclaims all liability and responsibility arising from any reliance placed on the content. Where the content includes any translated material, BMJ does not warrant the accuracy and reliability of the translations (including but not limited to local regulations, clinical guidelines, 
terminology, drug names and drug dosages), and is not responsible for any error and/or omissions arising from translation and adaptation or otherwise.

Open access This is an open access article distributed in accordance with the Creative Commons Attribution Non Commercial (CC BY-NC 4.0) license, which permits others to distribute, remix, adapt, build upon this work non-commercially, and license their derivative works on different terms, provided the original work is properly cited, appropriate credit is given, any changes made indicated, and the use is non-commercial. See: http://creativecommons.org/licenses/by-nc/4.0/.

\section{ORCID iD}

Elin Danielsen Lunde http://orcid.org/0000-0002-4058-953X

\section{REFERENCES}

1 Chugh SS, Havmoeller R, Narayanan K, et al. Worldwide epidemiology of atrial fibrillation: a global burden of disease 2010 study. Circulation 2014;129:837-47.

2 Hindricks G, Potpara T, Dagres N, et al. 2020 ESC guidelines for the diagnosis and management of atrial fibrillation developed in collaboration with the European association for Cardio-Thoracic surgery (EACTS). Eur Heart J 2021;42:373-498.

3 Ogilvie IM, Newton N, Welner SA, et al. Underuse of oral anticoagulants in atrial fibrillation: a systematic review. Am J Med 2010;123:638-45.

4 Lunde ED, Nielsen PB, Riahi S, et al. Associations between socioeconomic status, atrial fibrillation, and outcomes: a systematic review. Expert Rev Cardiovasc Ther 2018;16:857-73.

5 Thygesen LC, Daasnes C, Thaulow I, et al. Introduction to Danish (nationwide) registers on health and social issues: structure, access, legislation, and archiving. Scand J Public Health 2011;39:12-16.

6 Lynge E, Sandegaard JL, Rebolj M. The Danish national patient register. Scand J Public Health 2011;39:30-3.

7 Schmidt M, Schmidt SAJ, Sandegaard JL, et al. The Danish national patient registry: a review of content, data quality, and research potential. Clin Epidemiol 2015;7:449-90.

8 Kildemoes HW, Sørensen HT, Hallas J. The Danish national prescription registry. Scand J Public Health 2011;39:38-41.

9 Baadsgaard M, Quitzau J. Danish registers on personal income and transfer payments. Scand J Public Health 2011;39:103-5.

10 Jensen VM, Rasmussen AW. Danish education registers. Scand J Public Health 2011;39:91-4.

11 Pedersen CB. The Danish civil registration system. Scand J Public Health 2011;39:22-5.

12 Lévy S, Breithardt G, Campbell RW, et al. Atrial fibrillation: current knowledge and recommendations for management. working group on arrhythmias of the European society of cardiology. Eur Heart $J$ 1998;19:1294-320.

13 Fuster V, En L, Asinger RW. Acc / AHA / ESC practice guidelines full text ACC / AHA / ESC guidelines for the management of patients with atrial fibrillation a report of the American College of cardiology / American heart association Task force on practice guidelines and the Europe. European Heart Journal 2001;22:1852-923.

14 Fuster V, Rydén LE, Cannom DS, et al. ACC/AHA/ESC 2006 guidelines for the management of patients with atrial fibrillationexecutive summary. Circulation 2006;114:700-52.

15 , Camm AJ, et al, European Heart Rhythm Association, European Association for Cardio-Thoracic Surgery. Guidelines for the management of atrial fibrillation: the task force for the management of atrial fibrillation of the European Society of cardiology (ESC). Eur Heart J 2010;31:2369-429.

16 Camm AJ, Lip GYH, De Caterina R, et al. 2012 focused update of the ESC guidelines for the management of atrial fibrillation. Eur Heart $J$ 2012;33:2719-47.

17 Lip GYH, Nieuwlaat R, Pisters R, et al. Refining clinical risk stratification for predicting stroke and thromboembolism in atrial fibrillation using a novel risk factor-based approach: the Euro heart survey on atrial fibrillation. Chest 2010;137:263-72.

18 Statistics Denmark. FAMAEKVIVADISP [online]. Available: https:// www.dst.dk/da/Statistik/dokumentation/Times/familieindkomst/ famaekvivadisp [Accessed 26 Jan 2021].

19 Galobardes B, Shaw M, Lawlor DA, et al. Indicators of socioeconomic position (Part 1). J Epidemiol Community Health 2006;60:7-12.

20 Gadsbøll K, Staerk L, Fosbøl EL, et al. Increased use of oral anticoagulants in patients with atrial fibrillation: temporal trends from 2005 to 2015 in Denmark. Eur Heart J 2017;38:ehw658.

21 Christesen AMS, Vinter N, Mortensen LS, et al. Inequality in oral anticoagulation use and clinical outcomes in atrial fibrillation: a Danish nationwide perspective. Eur Heart J Qual Care Clin Outcomes 2018;4:189-99.

22 Ministry of Food, Agriculture and Fisheries. The Danish rural development programme 2007-2013 [online], 2012. Available: https://naturerhverv.dk/fileadmin/user_upload/NaturErhverv/Filer/ Tilskud/Projekttilskud/Landdistrikter/LDP_Rev_proposal_Consolid_ 2007-2013f.pdf [Accessed 23 May 2021].

23 Lunde ED, Joensen AM, Lundbye-Christensen S, et al. Socioeconomic position and risk of atrial fibrillation: a nationwide Danish cohort study. J Epidemiol Community Health 2020;74:7-13.

24 Pisters R, Lane DA, Nieuwlaat R, et al. A novel user-friendly score (HAS-BLED) to assess 1-year risk of major bleeding in patients with atrial fibrillation: the Euro heart survey. Chest 2010;138:1093-100.

25 Murphy NF, Simpson CR, Jhund PS, et al. A national survey of the prevalence, incidence, primary care burden and treatment of atrial fibrillation in Scotland. Heart 2007;93:606-12.

26 DeWilde S, Carey IM, Emmas C, et al. Trends in the prevalence of diagnosed atrial fibrillation, its treatment with anticoagulation and predictors of such treatment in UK primary care. Heart 2006;92:1064-70.

27 Meschia JF, Merrill P, Soliman EZ, et al. Racial disparities in awareness and treatment of atrial fibrillation: the reasons for geographic and racial differences in stroke (REGARDS) study. Stroke 2010;41:581-7.

28 Frewen J, Finucane $\mathrm{C}$, Cronin $\mathrm{H}$, et al. Factors that influence awareness and treatment of atrial fibrillation in older adults. QJM 2013;106:415-24.

29 Pathirana TI, Jackson CA. Socioeconomic status and multimorbidity: a systematic review and meta-analysis. Aust N Z J Public Health 2018;42:186-94.

30 Wehbe RM, Yadlapati A. Underuse of oral anticoagulants for nonvalvular atrial fibrillation: past, present, and future. Tex Heart Inst J 2016;43:287-90.

31 Svendsen MT, Bak CK, Sørensen K, et al. Associations of health literacy with socioeconomic position, health risk behavior, and health status: a large national population-based survey among Danish adults. BMC Public Health 2020;20:1-12.

32 Staerk L, Fosbøl EL, Gadsbøll K, et al. Non-vitamin K antagonist oral anticoagulation usage according to age among patients with atrial fibrillation: temporal trends 2011-2015 in Denmark. Sci Rep 2016;6:1-9.

33 Mårtensson S, Gyrd-Hansen D, Prescott E, et al. Socio-economic position and time trends in invasive management and case fatality after acute myocardial infarction in Denmark. Eur J Public Health 2016;26:146-52.

34 Statistics Denmark. HFFSP ("Forspaltekode til højst fuldførte uddannelse") [online]. Available: https://www.dst.dk/da/TilSalg/ Forskningsservice/Dokumentation/hoejkvalitetsvariable/hoejstfuldfoerte-uddannelse/hffsp [Accessed 23 May 2020].

35 Rix TA, Riahi S, Overvad K, et al. Validity of the diagnoses atrial fibrillation and atrial flutter in a Danish patient registry. Scand Cardiovasc J 2012;46:149-53.

36 Frost L, Andersen LV, Vestergaard P, et al. Trend in mortality after stroke with atrial fibrillation. Am J Med 2007;120:47-53. 